\title{
Unique case of dual gynecological malignancies co-existing with an uncommon thyroid lymphoma
}

\author{
Kellie Alleyne-Mike, Khadiga Elfadil Ahmed Mohammed, \\ Adriaan Leon van Wijk, Francois Cornelius Jacobus Botha
}

\begin{abstract}
Introduction: Synchronous primaries are uncommon in medical literature but pose many diagnostic and therapeutic challenges which require a multi-disciplinary approach. Case Report: A 64-year-old female was presented with a vulva lesion and bilateral inguinal lymphadenopathy. She was also noted to have a palpable abdominal growth and an enlarged neck mass. Blood evaluation yielded elevated CA-125 and thyroid stimulating hormone levels. All other tests were unremarkable. Computed tomography of the neck showed a large thyroid mass with bilateral lobe involvement and abdominal scanning showed a large cystic lesion causing right hydronephrosis and hydroureter. She was diagnosed with a primary vulva squamous carcinoma in a background of lichen sclerosus which was managed surgically. The patient also underwent laparotomy and was found to have a borderline ovarian malignancy. A neck biopsy yielded a thyroid lymphoma which was treated with chemotherapy. Prophylactic radiation to the groin was performed after multi-disciplinary consult. There was an excellent clinical response
\end{abstract}

Kellie R Alleyne-Mike ${ }^{1}$, Khadiga EA Mohamed ${ }^{1}$, Leon van Wijk$^{2}$, Francois CJ Botha ${ }^{3}$

Affiliations: ${ }^{1}$ Senior Resident, Radiation Oncology Department, Groote Schuur Hospital, Cape Town, South Africa; ${ }^{2}$ Consultant, Radiation Oncology Department, Groote Schuur Hospital, Cape Town, South Africa; ${ }^{3}$ Resident, Division of Pathology, Groote Schuur Hospital, Cape Town, South Africa.

Corresponding Author: Kellie Rozelle Alleyne-Mike, LE 33, Groote Schuur Hospital, Anzio Road, Observatory 7925, Cape Town, South Africa Ph: 27-21-791926866 or 868-7580192; Fax Number: 27-21-4485707; Email: kmike.tt@gmail. com

Received: 07 June 2013

Accepted: 11 July 2013

Published: 01 December 2013 to the treatment. Conclusion: This is the first report of concurrent vulva and ovarian pathology associated with a hematologic malignancy. Specific immunologic and genetic abnormalities have not yet been identified in literature which may link these supposedly random occurrences. However, the immune dysregulation associated with lichen sclerosus and its role in the pathogenesis of vulva squamous carcinoma is of interest in the background of a co-existing hematological malignancy, and an inherent compromised immune response. In addition, ongoing genetic research may yet elucidate a common link between these synchronous primaries.

Keywords: Synchronous primary malignancies, Thyroid lymphoma, Vulva cancer, Ovarian cancer

$* * * * * * * * *$

Alleyne-Mike KR, Mohamed KEA, van Wijk L, Botha FCJ. Unique case of dual gynecological malignancies co-existing with an uncommon thyroid lymphoma. International Journal of Case Reports and Images 2013;4(12):714-718.

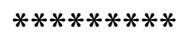

doi:10.5348/ijcri-2013-12-418-CR-12

\section{INTRODUCTION}

Synchronous primaries often pose unique management challenges. A careful analysis, discussion of patient and disease-related factors are required to guide appropriate management. It has been suggested that the upper female reproductive tract is an extension of the Mullerian system and thus carcinogenic exposure to this unithas the potential to produce multicentric lesions [1]. However, the lower female genital tract does not originate from the Mullerian 
system and the presence of synchronous primaries in both the upper and lower genital tract, as is presented in this case, cannot be explained by common embryonic origin. Further, a dual gynecological malignancy synchronously coexisting with a hematological malignancy is an unusual occurrence and will be discussed in view of the therapeutic challenges presented.

\section{CASE REPORT}

A 64-year-old female, with a medical history of hypertension, ischemic heart disease, and previous total abdominal hysterectomy for a benign indication, presented to the gynecology department with a six month history of a pruritic sore to the right labium minorus. Clinically, the lesion was $3 \mathrm{~cm}$ in diameter and extended partially across the midline with involvement of the clitoris. Two clinically palpable, $1 \mathrm{~cm}$ ipsilateral mobile inguinal lymph nodes were noted. On further clinical examination, an abdominal mass was palpated arising from the pelvis and extending upwards to above the umbilicus. Additionally, she had experienced progressive dysphagia for the previous three months. An enlarged, smooth thyroid gland with bilateral lobe involvement was present.

Investigations showed an elevated CA-125 558 $\mathrm{kU} / \mathrm{L}$ and $\mathrm{TSH} 14 \mathrm{mIU} / \mathrm{L}$. Full blood count, lactate dehydrogenase (LDH), renal and liver function tests were normal. HIV and syphilis serology were negative. Computed tomography (CT) scan of neck showed a massively enlarged thyroid gland with a maximum diameter of $9.4 \mathrm{~cm}$ (right lobe) and $9.1 \mathrm{~cm}$ (left lobe) (Figure 1). There was associated esophageal compression and the mass displaced the airway anteriorly without compromise. The CT scan of chest was negative for malignancy and CT scan of abdomen showed an enlarged liver and spleen as well as a central pelvic lesion extending into the abdomen (Figure 2A) causing right hydronephrosis and hydroureter (Figure 2B).

Biopsy of the vulva lesion was reported as a nonkeratinizing squamous cell carcinoma (Figure 3A) with associated lichen sclerosus, while that of the thyroid gland was consistent with a diffuse large B cell lymphoma (DLBCL) (Figure 3B-D). Repeated cytological aspiration (biopsies) of the inguinal nodes failed to confirm malignancy. Further staging investigations included a bone marrow biopsy which was clear.

The patient was discussed in a multi-disciplinary clinic with input from clinical oncology, gynecology, hematology, radiology, pathology and anesthesiology.

After extensive discussion, it was agreed to forgo surgical management of the groin due to concerns over potential morbidity which would delay commencement of chemotherapy. The patient underwent laparotomy during which a cystic left ovarian tumor was found. There was no ascites, peritoneal or omental nodules seen during the abdominal exploration. Peritoneal cytology was obtained in a standard manner, followed by bilateral salpingo- oophorectomy and an infracolic omentectomy. Surgical management of the vulva primary was done under the same anesthesia and consisted of a radical wide local excision. The postoperative course was unremarkable.

The histology of the vulvar lesion was of a moderately differentiated squamous cell carcinoma which measured $23 \mathrm{~mm}$ at its widest diameter. The depth of invasion was $6.2 \mathrm{~mm}$. The closest resection margin was $6.5 \mathrm{~mm}$. The stage was at least FIGO IB, though incomplete because of the unknown nodal status. The ovarian histology was of borderline serous cystadenocarcinoma, FIGO stage IA (Figure 3E-F).

Following surgery, the CA-125 level normalized. It was decided to start chemotherapy for the DLBCL concurrently with prophylactic radiation to the groin bilaterally because inguinal node dissection was not performed. Treatment was planned using $12 \mathrm{MeV}$ to deliver 45 Gy in 25 fractions (maximum depth of penetration $90 \%$ at $3 \mathrm{~cm}$ after measurement of lymph node depth was undertaken using CT imaging). The systemic therapy for the thyroid lymphoma, staged as IE, was with CHOP (cyclophosphamide, vincristine, prednisone and adriamycin) for a total of six cycles. Prior to chemotherapy, the patient's left ventricular ejection fraction (LVEF) was estimated with an equilibrium radionuclide angiocardiography (ERNA) to be $84 \%$. A follow-up ERNA did not indicate any LVEF compromise.

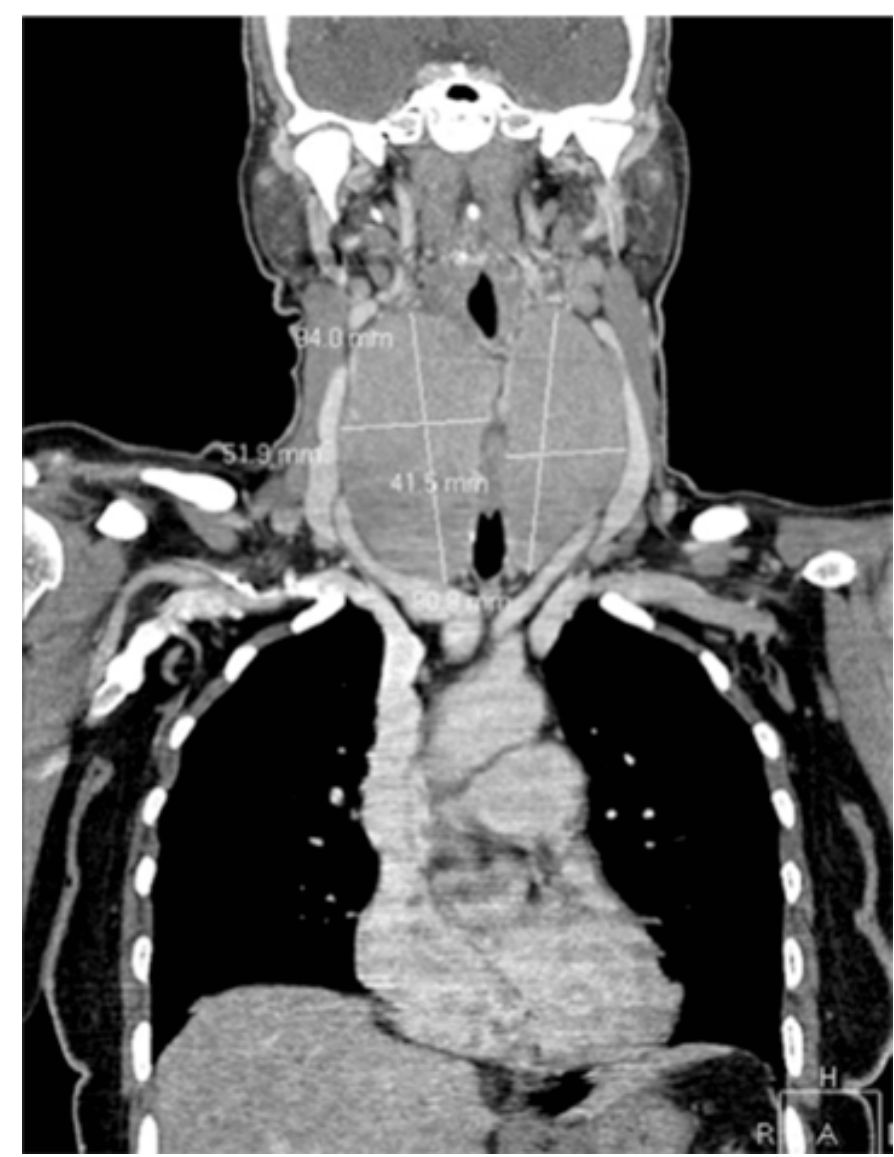

Figure 1: Coronal computed tomography of the chest and neck prior to chemotherapy showing massively enlarged thyroid with airway displacement. The right thyroid lobe measures 94x52 $\mathrm{mm}$ and the left measures 91x42 $\mathrm{mm}$. 


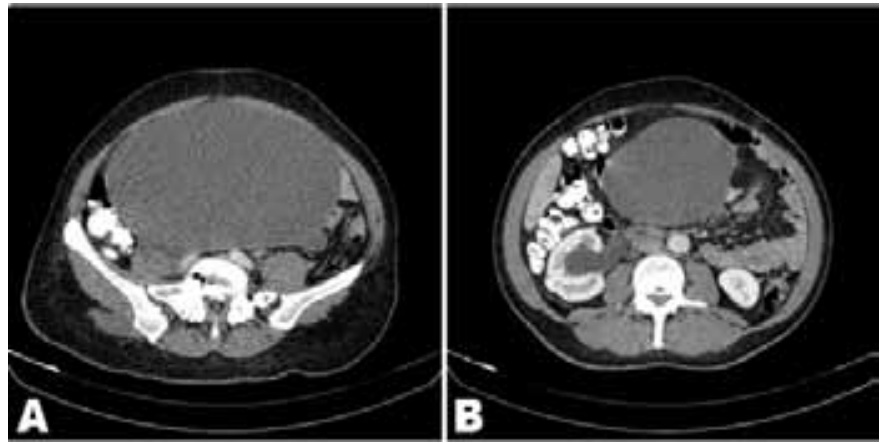

Figure 2: Axial computed tomography of the abdomen prior to surgery (A) showing a large well homogenated central pelvic lesion measuring 150x190x224 mm extending up into the abdomen, (B) with right hydronephrosis and hydroureter due to mass effect by the large abdominal mass.

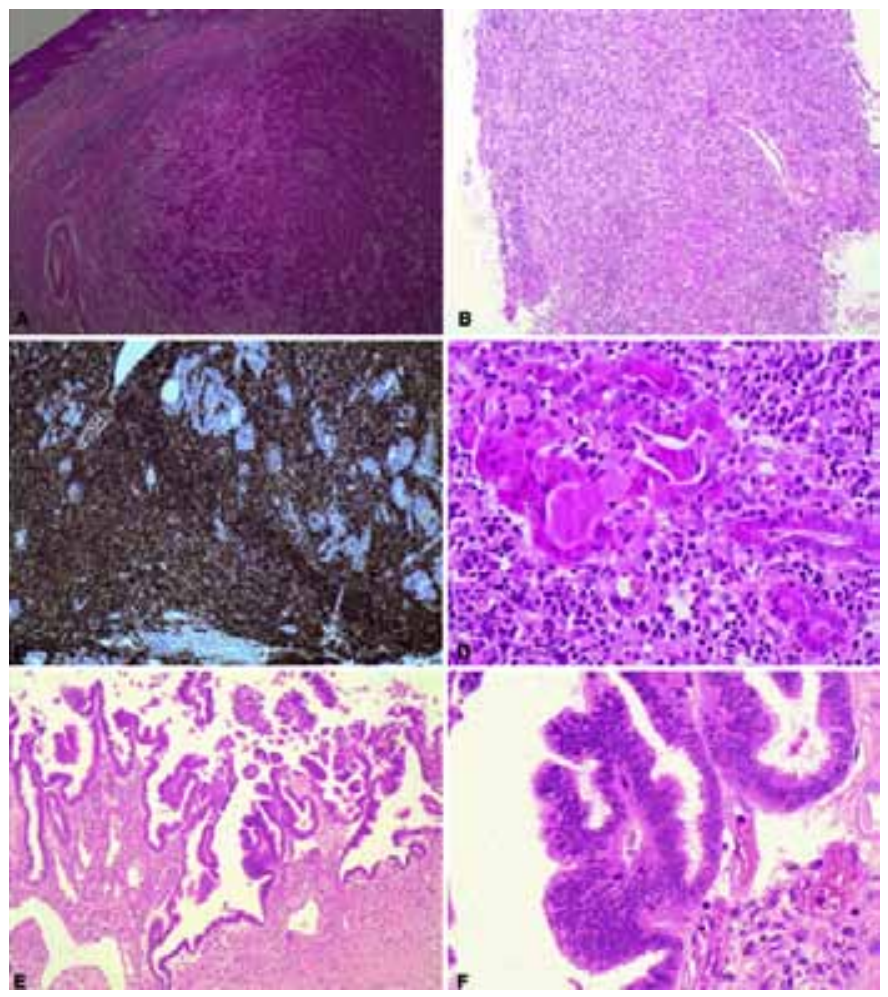

Figure 3: (A) Invasive moderately differentiated keratinizing squamous cell carcinoma with adjacent mild lichen sclerosis demonstrated by hyalinization of the papillary dermis and underlying chronic inflammation ( $\mathrm{H} \& \mathrm{E}$ stain, $\mathrm{x} 400$ ), (B) Core biopsy of the thyroid gland (H\&E stain, x100), (C) With a diffuse infiltrate of large atypical B-lymphocytes highlighted by CD-20 (H\&E stain, x200), (D) Scattered residual thyroid follicles (H\&E stain, x200), (E) Borderline serous tumor of the ovary with large papillary excrescences (H\&E stain, $\mathrm{x100),}$ and (F) Showing complex hierarchal branching and epithelial stratification (H\&E stain, x2OO).

\section{DISCUSSION}

In gynecologic oncology, the most frequently documented synchronous lesions are endometrial and ovarian malignancies [2]. Synchronous vulva and ovarian primaries are rare. The DLBCL is an aggressive malignancy which requires urgent chemotherapy. However, this patient's other lesions required surgical management and commencement of chemotherapy for the lymphoma would mean an extensive delay of the surgery. Thus, prompt surgical intervention was undertaken as the first course of action.

It was agreed to forgo surgical management of the groin as this has been shown to be associated with significant morbidity. In an article by Gaarenstroom et al. [3], almost $70 \%$ of the patients exhibited complications from the procedure, which is also the experience at the authors' institution. These problems are mostly wound breakdown and infection (among others). This risk of delayed wound healing would have impacted the urgent commencement of chemotherapy for DLBCL.

The main indication for adjuvant radiotherapy (RT) to the vulva at our institution is the resection margin status- positive or $<8 \mathrm{~mm}$. In this patient, all margins were $>6.5 \mathrm{~mm}$ (Lymphovascular invasion was not present but the depth of invasion was $6.5 \mathrm{~mm}$.) The closest excision margin of $6.5 \mathrm{~mm}$ was accepted as a reasonable compromise and adjuvant RT of the tumor bed was not performed.

Furthermore, RT to the vulva is often associated with moist desquamation which, coupled with impending cytotoxic chemotherapy for her primary lymphoma, would increase her risk of local infection, and cause unnecessary delays in treatment. Re-excision of the primary site would also have further delayed commencement of chemotherapy.

Concern over surgical complications associated with management of the groin prompted the consideration of prophylactic RT to the groin. A Cochrane analysis update by van der Velden suggested that although primary groin irradiation is inferior to primary groin surgery in terms of survival, the morbidity associated with primary surgery was significant [4]. Inadequate RT technique may have been the cause of worse outcome in the RT group. Petereit et al. argued that there was no difference in a three-year cause specific survival between primary RT and primary surgery to the groin but documented greater toxicity in the surgery group including infections in $44 \%$ and wound separation in $68 \%$ of patients [5]. An interesting study by Kalidas et al. measured the lymph node depths of patients with lower gynecological malignancies and found wide variations based on body habitus [6]. Of the 84 deep inguinal nodes measured, all were located at a depth of $>3 \mathrm{~cm}$. The study suggested that no substitute can be made for measurement of inguinal lymph nodes and that radiation treatment plans in previous studies were often suboptimal as generalizations were made concerning lymph node depth, which cannot be applicable to all patients.

Older patients should be initiated with the same dose intensity as young patients where possible with subsequent adjustments made depending on tolerance. Our patient, aged 64 years, had a good performance status and controlled co-morbidities, which prompted the use 
of CHOP at typical doses. At our institution rituximab is restricted to young, fit patients with early stage disease due to resource limitations, thus this was not a part of the regimen employed in our patient. She received six cycles of CHOP with one delay at cycle four due to neutropenia which did not require hospital admission. The patient is currently disease free from last eight months.

A specific mechanism has not been identified to link these individual entities. However, immunological and genetic abnormalities may be potential contributors to the pathogenesis of such synchronous neoplasms. Hu et al. commented that a compromised immune surveillance was inherent in DLBCL and that this could predispose such individuals to dual neoplasms [7]. Interestingly, the pathogenesis of squamous cell carcinoma from lichen sclerosus has been linked to immunologic dysregulation [8]. In addition, genetic factors such as RASSF2A, MGMT, TSP-1, p 53 and microsatellite instability have also been identified and may play a vital role in this process [9]. With regards to ovarian pathologies, syndromes genetically associated with BRCA1, BRCA2 or DNA repair genes such as hMSH2 and hMLH1 have been identified [10]. However, whether any parallels can be drawn to this situation is a subject for further research.

\section{CONCLUSION}

The findings outlined in this case are of unknown significance due to the rarity of the occurrence. This is the first documented presentation of such a case and thus may propel interest in further research into the genetics of multiple synchronous malignancies. The accumulation of case reports may ultimately be of benefit for the systematic analysis of multiple neoplasms as the reasons for their occurrence are likely multi-factorial.

\section{Author Contributions}

Kellie Alleyne-Mike - Conception and design, Acquisition of data, Analysis and interpretation of data, Drafting the article, Critical revision of the article, Final approval of the version to be published

Khadiga Elfadil Ahmed Mohammed - Analysis and interpretation of data, Critical revision of the article, Final approval of the version to be published

Adriaan Leon van Wijk - Analysis and interpretation of data, Critical revision of the article, Final approval of the version to be published

Francois Cornelius Jacobus Botha - Acquisition of data, Critical revision of the article, Final approval of the version to be published.

\section{Guarantor}

The corresponding author is the guarantor of submission.

\section{Conflict of Interest}

Authors declare no conflict of interest.

\section{Copyright}

(C) Kellie Alleyne-Mike et al. 2013; This article is distributed under the terms of Creative Commons attribution 3.0 License which permits unrestricted use, distribution and reproduction in any means provided the original authors and original publisher are properly credited. (Please see www.ijcasereportsandimages.com/ copyright-policy.php for more information.)

\section{REFERENCES}

1. Eisner RF, Nieberg RK, Berek JS. Synchronous primary neoplasms of the female reproductive tract. Gynecol Oncol 1989 Jun;33(3):335-9.

2. Zaino R, Whitney C, Brady MF, DeGeest K, Burger RA, Buller RE. Simultaneously detected endometrial and ovarian carcinomas--a prospective clinicopathologic study of 74 cases: a gynecologic oncology group study. Gynecol Oncol 2001 Nov;83(2):355-62.

3. Gaarenstroom KN, Kenter GG, Trimbos JB, et al. Postoperative complications after vulvectomy and inguinofemoral lymphadenectomy using separate groin incisions. Int J Gynecol Cancer 2003 JulAug;13(4):522-7.

4. van der Velden J, Fons G, Lawrie TA. Primary groin irradiation versus primary groin surgery for early vulvar cancer. Cochrane Database Syst Rev 2011 May 11;(5):CDo02224.

5. Petereit DG, Mehta MP, Buchler DA, Kinsella TJ. Inguinofemoral radiation of No, N1 vulvar cancer may be equivalent to lymphadenectomy if proper radiation technique is used. Int J Radiat Oncol Biol Phys 1993 Nov 15;27(4):963-7.

6. Kalidas H. Influence of inguinal node anatomy on radiation therapy techniques. Med Dosim 1995;20(4):295-300.

7. $\mathrm{Hu} \mathrm{XR}, \mathrm{Hu} \mathrm{YX}, \mathrm{Fu} \mathrm{HR}$, et al. Diffuse large B-cell lymphoma with concurrent gastric adenocarcinoma: case report and literature review. J Int Med Res 2011;39(5):2051-8.

8. Regauer S. Immune dysregulation in lichen sclerosus. Eur J Cell Biol 2005 Mar;84(2-3):273-7.

9. Gutierrez-Pascual M, Vicente-Martin FJ, LopezEstebaranz JL. Lichen sclerosus and squamous cell carcinoma. Actas Dermosifiliogr 2012 Jan;103(1):218.

10. Russo A, Calò V, Bruno L, Rizzo S, Bazan V, Di Fede G. Hereditary ovarian cancer. Crit Rev Oncol Hematol 2009 Jan;69(1):28-44. 
Access PDF of article on other devices

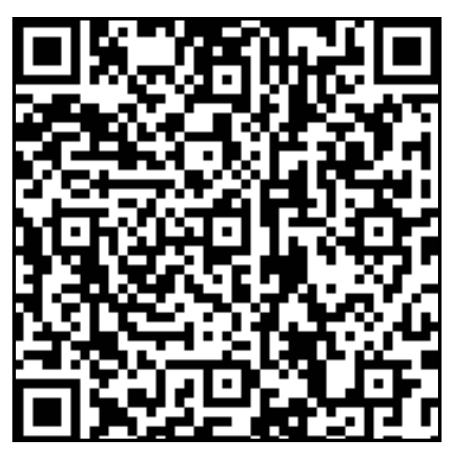

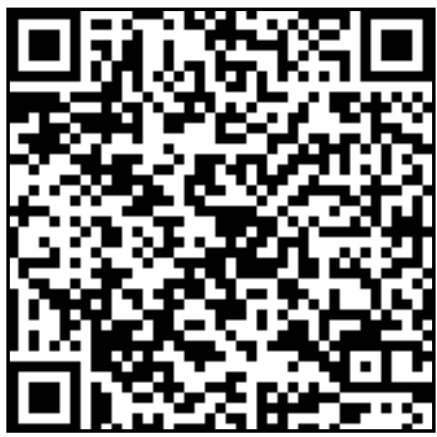

